Classification

Physics Abstracts

41.80Dd $-07.80+\mathrm{x}$

\title{
A simple UHV electron projection microscopy
}

\author{
Roger Morin, Alain Gargani and Francis Bel \\ CRMC2 $\left(^{\star}\right)$-CNRS, Campus de Luminy, Case 913, 13288 Marseille Cedex 09, France
}

(Received November 22, 1990; accepted December 28, 1990)

\begin{abstract}
Résumé. - On décrit un microscope électronique à projection de construction simple fonctionnant en ultra-vide. Des images de trous dans des membranes de carbone sont obtenues avec un grandissement allant jusqu'à 90000 . Sur de tels détails, cette microscopie électronique à projection apparait plus performante que la microscopie à balayage en termes de contraste, résolution et temps d'acquisition pour l'obtention d'une image.
\end{abstract}

\begin{abstract}
A simple electron projection microscope operating in ultra high vacuum is described. Images of holes in carbon membranes are obtained up to a magnification of 90000 . On such details, this electron projection microscopy appears superior than scanning electron microscopy in terms of contrast, resolution and acquisition time of an image.
\end{abstract}

\section{Introduction.}

In projection microscopy a magnified projection of an object is obtained by illuminating this object with a divergent beam of particules (Fig. 1). Though not new (Ref. [1]), this kind of microscopy, that we will call EPM (for electron projection microscopy) when a field emission tip is used as a source, appears quite promising by the introduction of the most recent technics borrowed from field-emission microscopy (FEM) and scanning tunneling microscopies (STM) and the use of "holly" carbon films (Ref. [2]). In projection microscopy the virtual size of the source is a key-point of the design (Ref. [3]). Therefore the use of electron sources of atomic size (Ref. [4]), for instance very sharp tips emitting electrons by field emission, is an important improvement. A specificity of this projection microscopy is to allow a very high contrast together with a good resolution. This is due mainly to the electron energy range that can be reached which is about 100 volts (this energy range is an important point compared for instance to projection microscopy using the focus of a standard scanning transmission electron microscope (Ref. [5]) where the energy is typically 3 orders of magnitude higher). Another feature is the absence of any lens (and associated aberrations) that excludes complex alignment problems and allows the obtention of

$\left(^{\star}\right)$ Laboratoire Propre du CNRS associé aux Universités d’Aix-Marseille 2 et 3. 


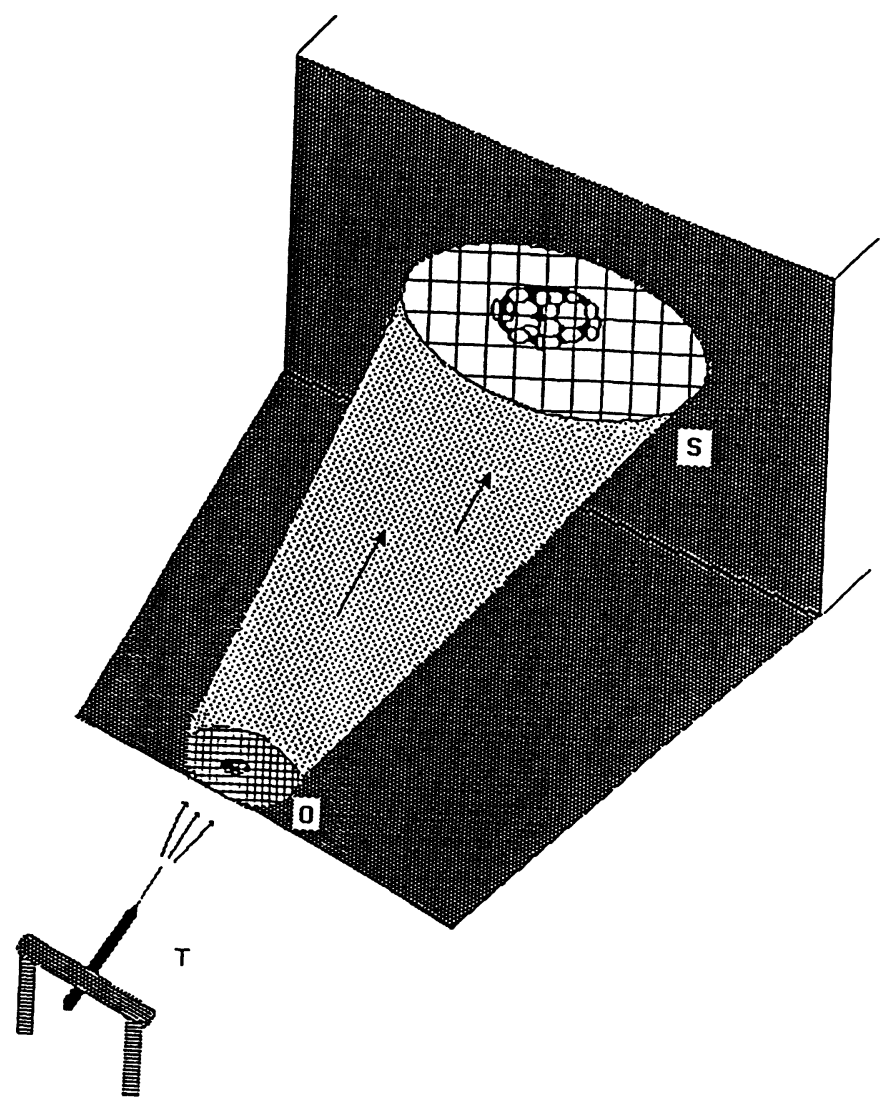

Fig. 1. - Projection microscopy principle.

highly magnified pictures without using any involved system. This aspect is particularly important when ultra high vacuum is required for instance.

In this paper, we describe a very simple design giving good images of carbon membranes and fibers. These images are compared to images of the same object obtained by standard scanning electron microscopy (SEM). This comparison allows to describe the main advantages of projection electron microscopy.

\section{Instrument design.}

Though the fact that the development of STM has caused available commercial micromanipulators, they are very expensive as well as non-directly suitable for some experiments. It is then interesting to sum up what are the requirements of a good manipulator for electron projection microscopy.

The tip to object distance has to be changed from macroscopic distances down to about 1000 Angstroms. At these last distances the magnification on a screen placed $1 \mathrm{~cm}$ away from the tip is already 100000 . A good stability of the tip position relative to the object position is required because vibrations would blur the pictures (the geometrical resolution is equal to the virtual size 


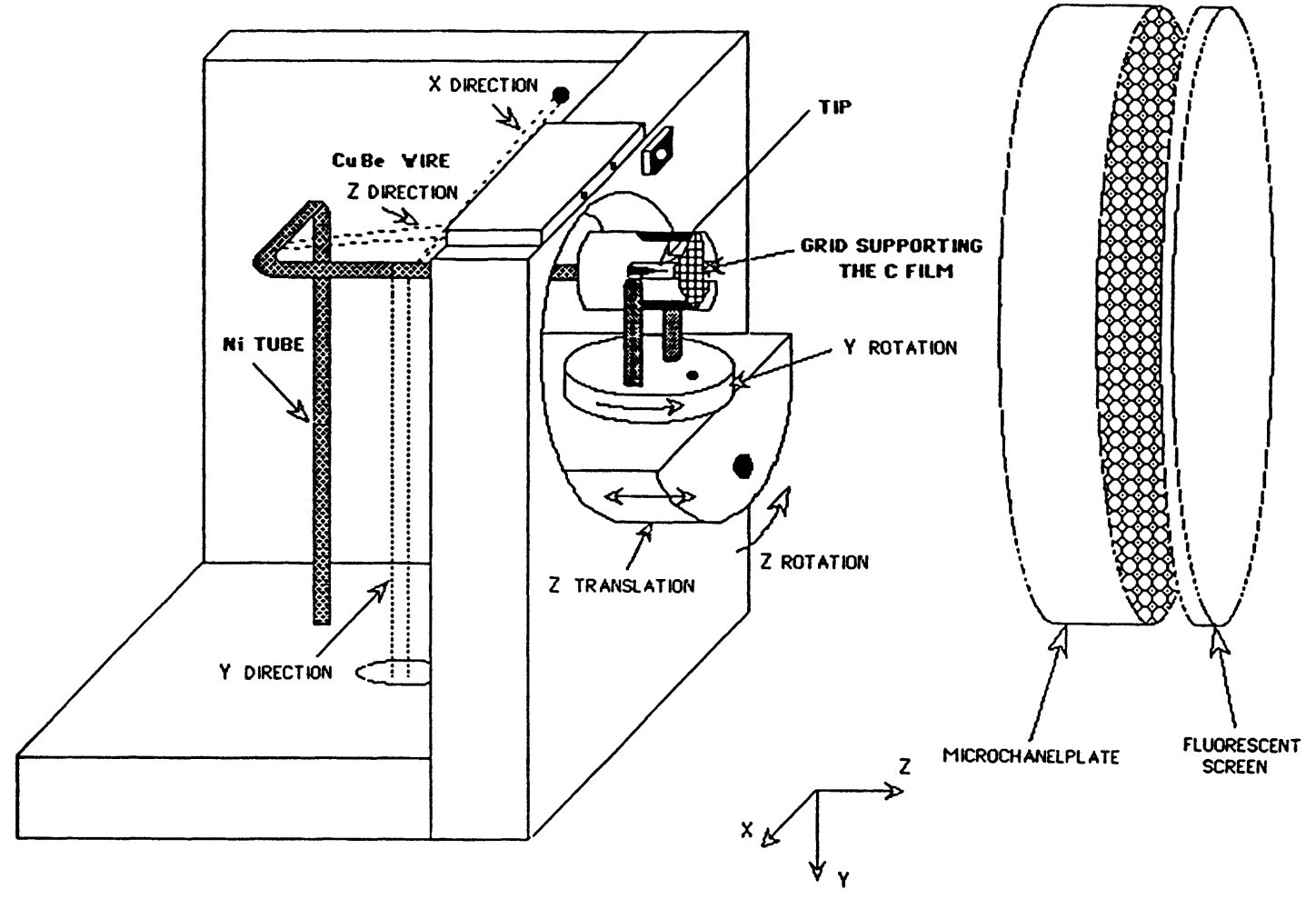

Fig. 2. - Scheme of the electron projection microscope.

of the source). A wide bandwith of displacements is not required as in scanning techniques, a picture being obtained as a whole for a given position of the tip.

The microscope uses thermal expansion of wires to move the object in front of a (110) oriented tungsten tip prepared by electrochemical etching. The microscope is shown in figure 2 . The manipulator is made of $\mathrm{Ni}$ tubes bent by 3 orthogonal $\mathrm{Cu}-\mathrm{Be}$ wires which are previously tightened. The tip is introduced inside the tube using fences made in the tube walls (the tip holder allows 2 rotations and one translation that permit a rough positioning of the tip. A cap holding the object (a carbon film supported by a copper grid) is introduced into the tube and approached to a distance of about $50 \mu \mathrm{m}$ from the tip using a micrometric pusher and optical microscope control (Fig. 3). Joule heating of the wires allows 3 dimensional displacement of the carbon foil. Electron intensity is detected using a $4 \mathrm{~cm}$ diameter channelplate phosphor screen assembly fixed $2.5 \mathrm{~cm}$ from the tip. The whole system is hung with viton links on a $100 \mathrm{CF}$ flange supporting also electrical feedthroughs. This flange is part of a ultra high vacuum chamber and pictures of the phosphor screen can be taken using video or photographic cameras.

\section{Results and discussion.}

The results described hereafter have to be considered as preliminary in a new domain and observations with well established microscopies will be helpful to understand the main features of EPM. 


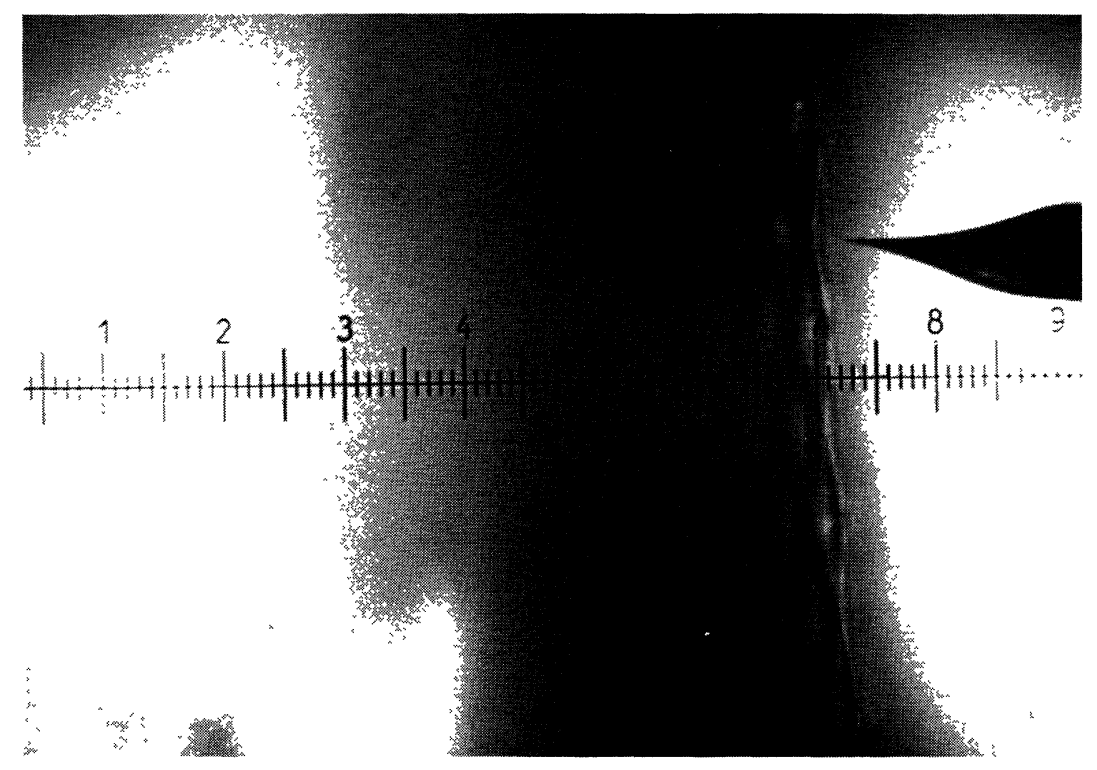

Fig. 3. - Optical micrograph of the tip in front of the carbon membrane. The tip to membrane distance is $50 \mu \mathrm{m}$.

Due to the design of the manipulator, the initial magnification is quite big (400). This magnification can be measured in situ from the projection of a $30 \mu \mathrm{m} \mathrm{Cu}$ wire which constitutes the grid (Fig. 4). It corresponds roughly to the ratio of the tip to screen distance $(2.5 \mathrm{~cm})$ to the tip to grid distance $(50 \mu \mathrm{m})$ as observed by optical microscopy. This means that, for this magnification, the positions of the real source and of the virtual one are the same. By side moving the grid it is also possible to determine the size of the region which can be observed which is a square corresponding to about 4 meshes ( $200 \mu \mathrm{m}$ in linear dimension). The upper limitation mainly comes from the emission of light from the heated wire that produces spurious signals on the screen.

Two sets of images taken with EPM and corresponding pictures of the same region taken with a scanning electron microscope Jeol 35C are shown in figures 5 and 6. SEM pictures were taken after EPM pictures. The localization of the interesting meshes is quite difficult because of the ignorance of the mesh position on the grid, and these meshes were found only after observing many others and comparing with EPM pictures. These images permit to understand very directly the main features of EPM.

3.1 CONTRAST AND RESOLUTION. - One striking difference between EPM and SEM pictures lies in the contrast which is much more important with EPM. SEM requires to work with a largely opened condenser in order to get a good enough contrast. This is likely due to the low secondary emission coefficient of carbon. But the excellent contrast obtained with this projection microscopy also comes from the very low energy used with EPM (100 volts) which is the energy range where the mean free path of electrons in many materials is minimal.

This contrast requirement has an important impact on the resolution of SEM on this kind of objects. This is illustrated in figure $6 \mathrm{i}$ compared to figure $6 \mathrm{c}$ where a small hole $(100 \mathrm{~nm})$ is clearly resolved in EPM whereas it is hardly seen with SEM. This shows that the potential resolution of 


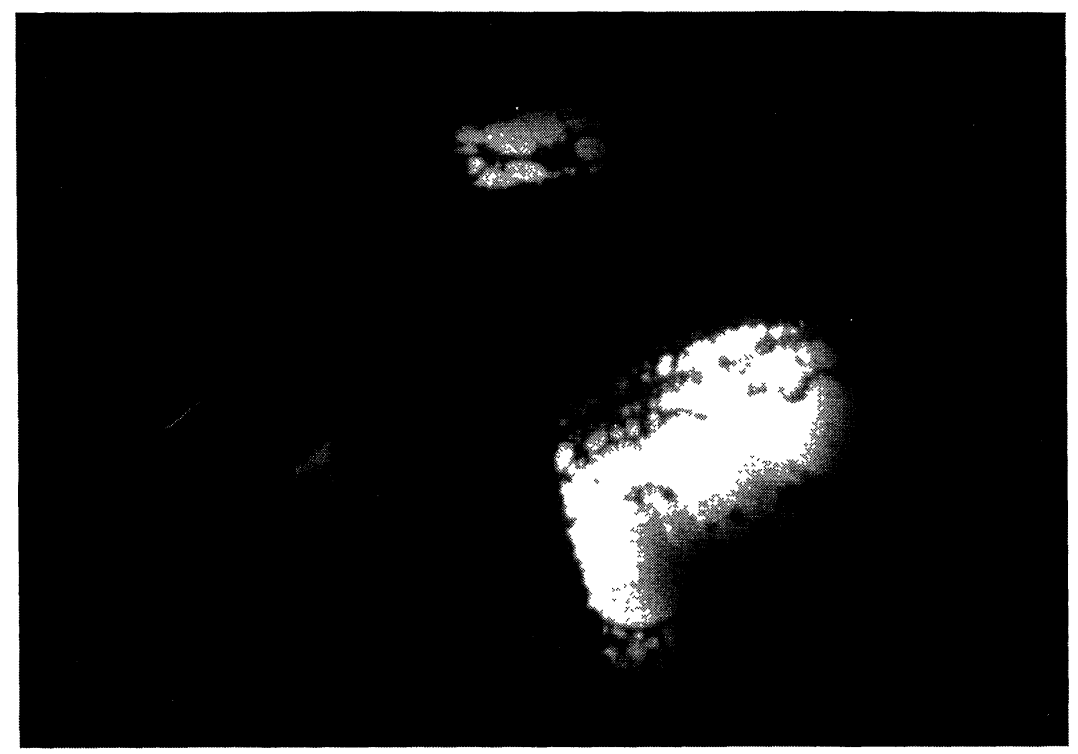

Fig. 4. - Projection electron micrograph showing the $\mathrm{Cu}$ wire ( $30 \mu \mathrm{m}$ in diameter) of the grid supporting the $\mathrm{C}$ membrane.

the technique is below a few hundred Angstroms as already reported in reference [2] by a different way of evaluation.

3.2 SPEED. - Another difference is the image acquisition time which is of $1 / 10$ second with EPM whereas it is 100 seconds with SEM. Significant decrease in this time could even be expected by working at higher current as long as the detector does not saturate. Apart of detector or object limitations, an increase of the total intensity emitted by the tip of 6 orders of magnitude is possible (the tip current is about a few pA in the present experiment and intensities of a few $\mu \mathrm{A}$ can easily be drawn from field emission tips). These considerations anticipate the use electron projection microscopy for the observation of fast dynamical processes.

3.3 MAgNifiCATION. - With this simple design it has been possible to work up to a magnification of about 90000 as hown in figure $5 \mathrm{~h}$. Magnification value is determined from SEM image comparison up to figures $5 \mathrm{~g}$ and then by comparison of figure $5 \mathrm{~g}$ and $5 \mathrm{~h}$. At the highest magnifications, the tip is $0.28 \mu \mathrm{m}$ above the carbon membrane and the stabilization of its position above a given detail takes some time. The node between the three filaments was destroyed when the tip touched it by accident and that is the reason why it cannot be seen on the SEM images taken afterwards. The tip was not destroyed during this event but the emission changes in such a way that this region was not anymore reachable in high magnification projection microscopy preventing any EPM observation of the damage.

3.4 MiscellaneOUS. - From the comparison of SEM and EPM micrographs it is clear that the observed objects appear quite similar but not identical. Probably most of the differences come from the fact that EPM gives a projection from a point and not an orthogonal projection (as SEM gives for these flat and thin objects) and significant differences may appear even for simple two 


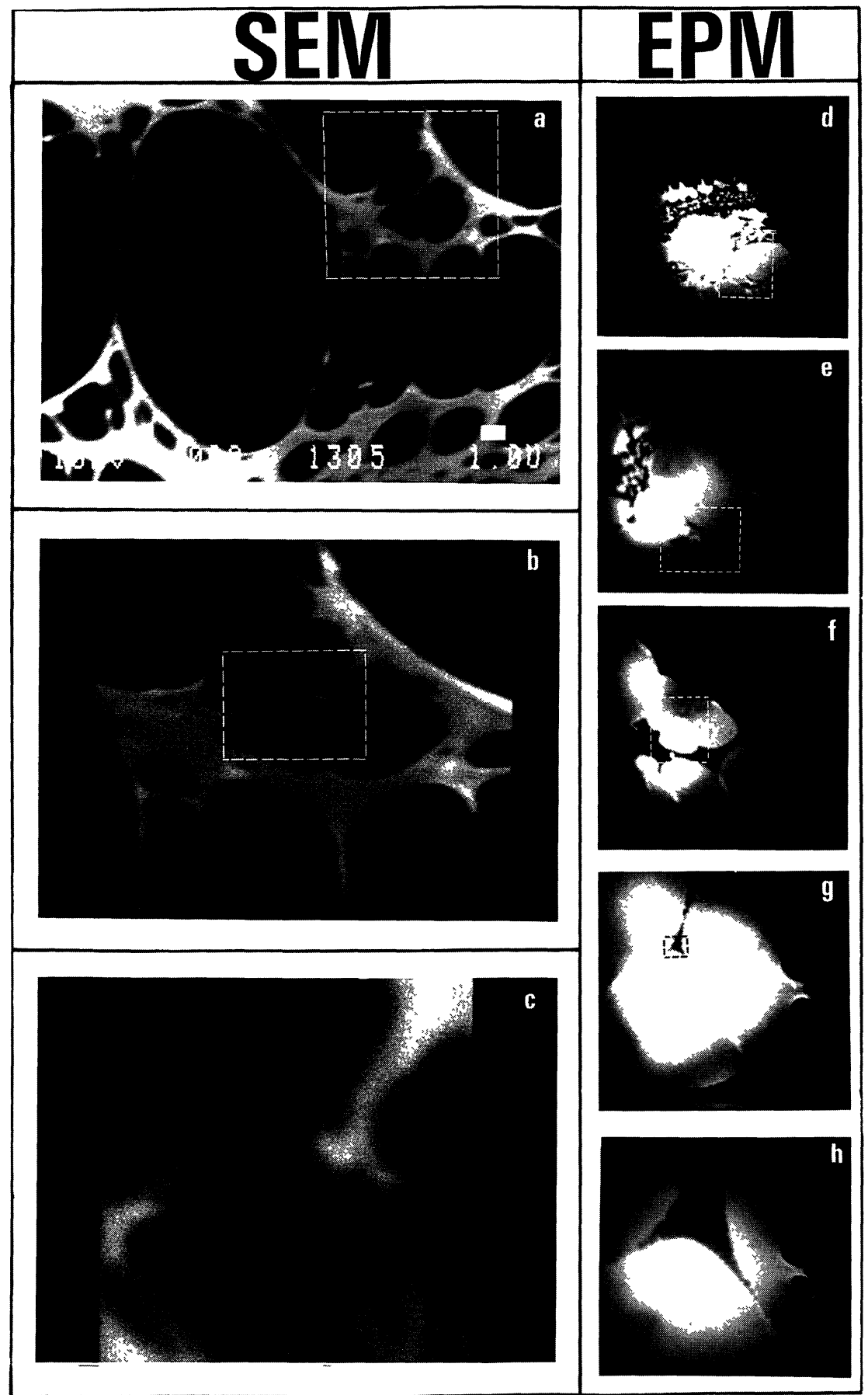

Fig. 5. - Scanning electron micrographs (SEM) (energy of the primary beam is $10 \mathrm{kV}$ ) and corresponding projection electron micrographs (EPM) of the same region of the carbon membrane. With each technique, a frame shows the region which is magnified on the next micrograph. The node between the 3 filaments (photo $\mathrm{c}$ ) was destroyed by the tip by accident during the projection experiment. The scale is indicated on a. From d to h electron energy varies from $120 \mathrm{eV}$ down to $35 \mathrm{eV}$. The respective magnification and tip to sample distance are: d: 400,62 $\mu \mathrm{m}$; e: 1000,25 $\mu \mathrm{m}$; f: 4000,6 $\mu \mathrm{m} ; \mathrm{g}: 12000,02 \mu \mathrm{m} ; \mathrm{h}: 90000,28 \mu \mathrm{m}$. 


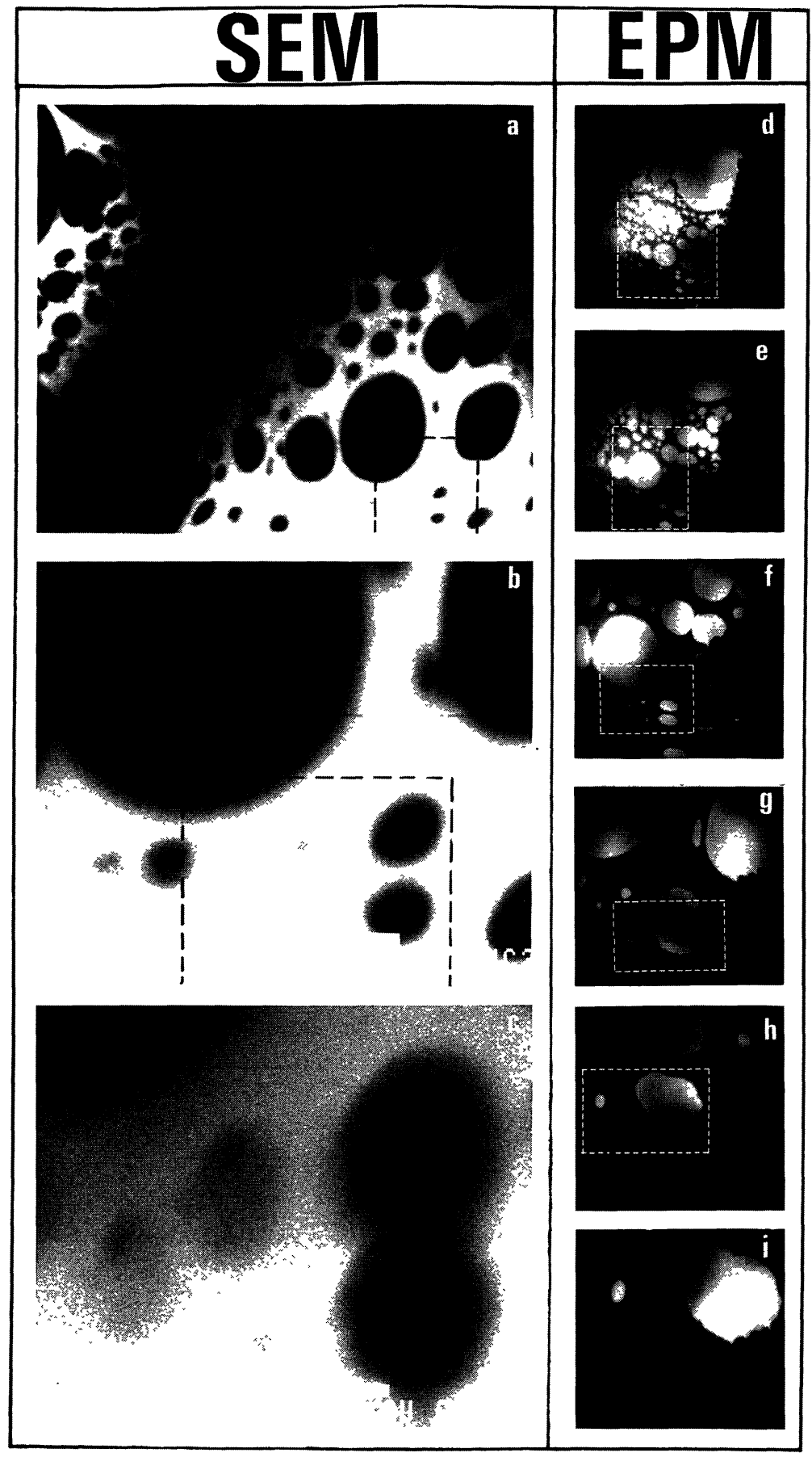

Fig. 6. - Scanning electron micrographs (SEM) (energy of the primary beam is $10 \mathrm{kV}$ ) and corresponding projection electron micrographs (EPM) of the same region of the carbon membrane. With each technique, a frame shows the part of the micrograph which is magnified on the next micrograph. The scale is indicated on $b$ and $c$. From d to e electron energy varies from $320 \mathrm{eV}$ down to $180 \mathrm{eV}$. The respective magnification and tip to sample distance are: d: $400,62 \mu \mathrm{m}$; e: $650,38 \mu \mathrm{m}$; f: $1300,19 \mu \mathrm{m} ; \mathrm{g}: 2800,9 \mu \mathrm{m}$; h: $4200,6 \mu \mathrm{m}$, i: $7600,3.3 \mu \mathrm{m}$. 
dimensional objects; for instance the general appearance of a circle in EPM would be an oval (even not an ellipse). Therefore we will not talk of distorsion for this effect.

One strange fact is that some filaments are dark whereas some other filaments are bright. We checked by biasing the front face of the channelplate that this is not correlated with the emission of electrons (which should in that case be emitted in the same direction as the primary ones, which is not the case of true secondaries). It is possible that some filaments are bright because of the curvature of the electron trajectories in the vicinity of the filaments due to transverse fields i.e. in the plane of the carbon membrane. This effect is known and prevents to observe any isolated small object with a high magnification because in that case the transverse field on the object is too high (Ref. [6]). In this context, it appears quite interesting to use tips which are very sharp because most of the voltage drop takes place in the vicinity of the tip apex and the field on the object is then reduced. In the present case, by the fact that the bright appearence of some filaments is magnification independent means that this electrostatic deflection is likely related to some charging effect of those filaments. A good indication of this is that charging effects, producing sudden shifts of the image, are observed in SEM on the same samples.

The damage produced using such a microscopy is difficult to determine presently. The only experimental fact is that one filament was observed with a magnification of about 100000 without noticeable image change during about 10 minutes. For comparison, some degradation on the same sample can be observed after a few minutes observation at a similar magnification using a $100 \mathrm{kV}$ transmission microscope Jeol 100C (Ref. [7]).

\section{Conclusion and outlook.}

We have built a simple electron projection microscope operating in ultra high vacuum and described the main features of projection microscopy by looking the carbon membranes with this technique and with classical scanning electron microscopy. On these objects, projection electron micrographs are obtained with a high contrast coupled with a good lateral resolution ( $<$ a few $10 \mathrm{~nm})$ ) in a 1000 times shorter time than corresponding scanning electron micrographs. This low energy electron projection microscopy then appears quite promising in the direction of observing thin samples made of light materials with which high energy electron beams usually give a weak contrast as well as a possible way, due to the high electron intensity, to observe some dynamical processes.

\section{Acknowledgements.}

We would like to dedicate this paper to the memory of the late Werner Stocker, IBM Zürich, with whom one of us (R.M.) began to work on projection microscopy using field emission sources. He also deeply appreciates the continuous interaction with his colleagues and friends, Hans-Werner Fink and Heinz Schmid, IBM Zürich. In the CRMC2, we specially thank Francis Beaumond for the machining work, Francis Quintric for the photographic part and Serge Nitsche for his help in microscopy. This work is partially supported by a Joint Project Agreement between IBM Research Laboratory Zürich and the CRMC2-CNRS. 


\section{References}

[1] Boersch H., Z. Tech. Phys. 12 (1939) 346;

MORTON G.A. and RAMBERG E.G., Phys. Rev. 56 (1939) 705.

[2] STOCKER W., FINK H.-W. and MORIN R., Ultramicroscopy 31 (1989) 379-384.

[3] See for instance FERT S., Traité de Microscopie Electronique (Hermann, Paris) 1961, pp. 343-349.

[4] Fink H.-W., Phys. Scr. 38 (1988) 260.

[5] ZEITLER E. and THOMSON M.G.R., Opticks 31 (1970) 258-280 and 359-366.

[6] Melmed A., Appl. Phys. Lett. 12 (1968) 100-102. PiQuet A., Roux H., Vu thien binh, Uzan R. et DRECHSLER M., Rev. Phys. Appl. 6 (1971) 105-109.

[7] NITSCHE S., private communication. 